\title{
Bioprosthetic valve thrombosis: What we know and what we need to know
}

\section{ABSTRACT}

Bioprosthetic valve thrombosis account for $11 \%$ of all reoperations for bioprosthetic valve dysfunction, and preoperative diagnosis can be made based on echocardiographic features. Early reoperation can be prevented if BPVT is identified and treated preoperatively. (J Thorac Cardiovasc Surg 2016;152:975-8)
\end{abstract}

Alexander C. Egbe, MD, MPH, ${ }^{a}$ Heidi M. Connolly, MD, FACC, ${ }^{a}$ and Hartzell V. Schaff, MD, FACC ${ }^{b}$

\begin{tabular}{l} 
Pex \\
Alexander C. Egbe, MD, MPH \\
Central Message \\
Bioprosthetic valve thrombosis is not an un- \\
commmon cause of bioprosthetic dysfunction. \\
It should be considered during evaluation for \\
prosthetic dysfunction. \\
Perspective \\
Preoperative diagnosis of bioprosthetic valve \\
thrombosis can be made based on echocardio- \\
graphy. Further research is required to delineate \\
its pathophysiology, and the optimal strategy \\
for the treatment and prevention of this disease. \\
See Editorial Commentaries page 978 \\
and 981. \\
\hline
\end{tabular}

The predominant mechanism of bioprosthetic valve dysfunction is structural deterioration. ${ }^{1,2}$ The risk of bioprosthetic valve thrombosis (BPVT) is unknown but generally considered very low. A recent study reported in the December 2015 issue of the Journal of American College of Cardiology $y^{3}$ has challenged this paradigm by demonstrating that BPVT is not an uncommon cause of prosthetic valve dysfunction. Here we review important findings from this recent study, and

From the Departments of ${ }^{\mathrm{a} C a r d i o v a s c u l a r}$ Diseases and ${ }^{\mathrm{b}}$ Cardiovascular Surgery, Mayo Clinic, Rochester, Minn.

Received for publication March 8, 2016; revisions received April 4, 2016; accepted for publication April 15, 2016; available ahead of print May 25, 2016.

Address for reprints: Hartzell V. Schaff, MD, FACC, Department of Cardiovascular

Surgery, Mayo Clinic, 200 First St SW, Rochester, MN 55905 (E-mail: schaff@ mayo.edu).

$0022-5223 / \$ 36.00$

Copyright (C) 2016 by The American Association for Thoracic Surgery

http://dx.doi.org/10.1016/j.jtcvs.2016.04.049 highlight unresolved questions and areas of future research in BPVT.

\section{HOW COMMON IS BPVT?}

Egbe and colleagues ${ }^{3}$ identified bioprosthetic valve thrombosis in 46 out of 397 (11\%) bioprosthetic valves explanted at Mayo Clinic. In contrast to the misconception that BPVT is a perioperative phenomenon, this study revealed that $65 \%$ of all reoperations for BPVT occurred more than 1 year after implantation, and up to $15 \%$ of these reoperations occurred more than 5 years after the initial implantation. The authors estimated the incidence of BPVT as $1 \%$ based on 3161 patients with an implanted bioprosthetic valve who underwent follow-up echocardiography at their institution.

Although this study highlights the role of BPVT as an important cause of prosthetic valve dysfunction requiring reoperation, the incidence of BPVT in the community is unknown. The $1 \%$ incidence reported in this study was 


\section{Abbreviation and Acronym \\ $\mathrm{BPVT}=$ bioprosthetic valve thrombosis}

based solely on a subset of the cohort of patients who had follow-up echocardiography. Unfortunately, almost one-half of the patients who underwent bioprosthetic valve implantation within the study period were excluded from the analysis because of a lack of follow-up echocardiograms available for review, and thus the true incidence of BPVT cannot be ascertained. Furthermore, the report of Egbe and colleagues was based on a single tertiary center experience, and the demographics of that patient population might not be representative of the general population with valvular heart disease owing to referral bias.

The second unresolved epidemiologic question is the timing of BPVT occurrence. This study used the "time to explantation" as surrogate for "time to thrombosis." Because the interval from the occurrence of BPVT thrombosis to reoperation is unknown, it is logical to assume that substituting the time to explantation for time to thrombosis will result in overestimation. A different study design is needed to determine BPVT incidence and the timing of BPVT occurrence.

The occurrence of BPVT is not restricted to surgically implanted bioprosthetic valves, but has also been observed after transcatheter aortic valve replacement (TAVR). A recent study in reported reduced leaflet motion in 22 of 55 patients $(40 \%)$ after TAVR. ${ }^{4}$ Restoration of leaflet motion was noted in all patients who received anticoagulation, suggesting that the bioprosthetic dysfunction observed in $40 \%$ of that cohort was due to BPVT. Furthermore, Del Trigo and associates ${ }^{5}$ observed deterioration in the hemodynamic function of transcatheter valves $(>10 \mathrm{mmHg}$ increase in gradient) in $4.5 \%$ of their patients over a mean follow-up of 20 months. ${ }^{5}$ In a multivariate analysis, failure to anticoagulate patients at hospital discharge was associated with an increased risk of valve dysfunction (hazard ratio, 3.35), lending additional support to thrombosis as a cause of valve dysfunction in some patients.

\section{HOW DO WE DIAGNOSE BPVT?}

In this study, the diagnosis of BPVT was considered in only 6 of 45 patients $(13 \%)$ who underwent transesophageal echocardiography. A significant proportion of the patients with BPVT were misdiagnosed as having structural failure and referred for reoperation. This attests to the low level of awareness of the existence of BPVT and the lack of well-defined diagnostic criteria.

The authors have proposed a BPVT diagnostic model based on echocardiography characteristics, including a $50 \%$ increase in prosthetic gradient within 5 years of implantation, increased cusp thickness, and abnormal cusp mobility. The presence of all 3 echocardiographic features reliably diagnosed BPVT with a sensitivity of $72 \%$ and a specificity of $90 \%$ when applied to the 138 patients included in the study. It is important to note that the performance of this diagnostic model was tested in highly selected cohort that underwent reoperation for severe bioprosthetic valve dysfunction. Perhaps this diagnostic model may be less predictive in other populations.

Routine annual echocardiography is not recommended within the first 5 years of bioprosthetic valve implantation. ${ }^{6}$ This recommendation is based on the assumption that the risk of structural failure (ie, cusp calcification or tear), which was thought to be the sole mechanism of bioprosthetic valve dysfunction, was very low within the first 5 years after valve implantation. This study shows that $85 \%$ of cases of BPVT occurred within the first 5 years, the period during which routine echocardiography is not recommended.

The occurrence of BPVT resulting in significant prosthetic dysfunction may be signaled by abnormal physical examination findings that should prompt echocardiographic examination, but clinical evaluation of progressive valve dysfunction is not completely reliable. If the objective is prompt identification of BPVT and early initiation of anticoagulation therapy, then reliance on abnormal physical examination findings as a prerequisite for echocardiography might not be the most effective surveillance strategy. In addition to transthoracic and transesophageal echocardiography, the use of other complementary imaging modalities, such as computed tomography, could be very effective in identifying subtle BPVT, as has been demonstrated in a study of bioprosthetic dysfunction after transcatheter aortic valve replacement. ${ }^{4}$

\section{HOW DO WE TREAT BPVT?}

In an initial study from the Mayo Clinic group, ${ }^{7} 14$ of 15 patients $(93 \%)$ with a presumed diagnosis of BPVT responded to anticoagulation therapy with warfarin and subsequently avoided reoperation. Several other case series, including studies of BPVT in transcatheter valves, have reported similar findings. ${ }^{8-11}$

A response to anticoagulation therapy for BPVT is usually observed within 4 to 12 weeks after the initiation of therapy. ${ }^{7-9}$ The optimal duration for trial of anticoagulation therapy, and the most effective anticoagulant (vitamin K antagonist vs novel oral anticoagulant) remain unknown, however.

\section{HOW DO WE PREVENT BPVT?}

The American College of Cardiology/American Heart Association and European Society of Cardiology guidelines do not recommend anticoagulation for bioprosthetic valves beyond the first 3 months after implantation. ${ }^{12,13}$ In the study by Egbe and colleagues, ${ }^{3}$ perioperative anticoagulation data were available for 63 patients $(46 \%)$, 
and the use of guideline-directed perioperative anticoagulation in 37 of these 63 patients $(59 \%)$ demonstrated no protective effect against BPVT occurrence. Antiplatelet therapy was also ineffective for BPVT prophylaxis, considering that almost all the patients were receiving antiplatelet therapy at the time of BPVT diagnosis. The authors showed that BPVT was not a perioperative event, and also suggested that the current guideline-directed perioperative prophylaxis might not be effective in preventing BPVT.

A large observational study of 4075 cases of aortic valve replacement from the Danish National Patient Registry clearly showed that discontinuation of anticoagulation within 6 months after surgical aortic valve replacement was associated with an increased risk of thromboembolic complications and cardiovascular death. ${ }^{14}$ These data, in addition to findings of early valve dysfunction, argue strongly for early anticoagulation with coumadin in all patients with a bioprosthetic heart valve.

Making evidence-based recommendations for the prevention of BPVT requires better risk stratification. Egbe and colleagues $^{3}$ identified the presence of paroxysmal atrial fibrillation (but not persistent atrial fibrillation) and inadequate anticoagulation (defined as international normalized ratio $<2.0$ ) as risk factors for BPVT. It is also noteworthy that more than one-half of the patients with BPVT did not have paroxysmal atrial fibrillation or an indication for anticoagulation at the time of diagnosis, further suggesting the presence of other unidentified clinical, demographic, and possibly prosthetic risk factors for BPVT.

The role of prosthesis type in the etiology of BPVT remains unclear. A previous study by Brown and colleagues ${ }^{15}$ reported 8 cases of aortic BPVT in porcine valves, none of which occurred in pericardial valves. In the study by Egbe and colleagues, although porcine valves were the predominant prosthesis type, pericardial valves accounted for 8 of the $46(18 \%)$ cases of BPVT. Thus, the presence of a porcine valve was not a predictor of BPVT in that study.

\section{FUTURE PERSPECTIVES}

Highlights of the present study can be summarized as follows:

- BPVT is not an uncommon cause of BPVD and is often unrecognized.

- BPVT is not a perioperative complication; it can occur up to several years after implantation.

- Preoperative diagnosis is feasible using echocardiographic features of BPVT.

- Most cases of BPVT occur within 5 years of implantation; thus, the current valvular heart disease guideline recommendations against routine echocardiography within the first 5 years of valve implantation should be revisited.
Some important questions remain unanswered, including:

- What is the incidence of BPVT?

- What is the best screening modality?

- What are other clinical and demographic risk factors for BPVT?

- What is the optimal duration of perioperative anticoagulation to prevent BPVT?

- What is the role of the novel oral anticoagulants in the treatment of BPVT?

Answers to these questions will come from populationbased prospective studies.

\section{Conflict of Interest Statement}

Authors have nothing to disclose with regard to commercial support.

\section{References}

1. Marchand M, Aupart M, Norton R, Goldsmith IR, Pelletier C, Pellerin M, et al Twelve-year experience with Carpentier-Edwards PERIMOUNT pericardial valve in the mitral position: a multicenter study. J Heart Valve Dis. 1998;7:292-8.

2. Hammermeister KE, Sethi GK, Henderson WG, Oprian C, Kim T, Rahimtoola S. A comparison of outcomes in men 11 years after heart-valve replacement with a mechanical valve or bioprosthesis. Veterans Affairs Cooperative Study on Valvular Heart Disease. N Engl J Med. 1993;328:1289-96.

3. Egbe AC, Pislaru SV, Pellikka PA, Poterucha JT, Schaff HV, Maleszewski JJ, et al. Bioprosthetic valve thrombosis versus structural failure: clinical and echocardiographic predictors. J Am Coll Cardiol. 2015;66:2285-94.

4. Makkar RR, Fontana G, Jilaihawi H, Chakravarty T, Kofoed KF, de Backer O, et al. Possible subclinical leaflet thrombosis in bioprosthetic aortic valves N Engl J Med. 2015;373:2015-24.

5. Del Trigo M, Muñoz-Garcia AJ, Wijeysundera HC, Nombela-Franco L, Cheema AN, Gutierrez E, et al. Incidence, timing, and predictors of valve hemodynamic deterioration after transcatheter aortic valve replacement: multicenter registry. J Am Coll Cardiol. 2016;67:644-55.

6. Zoghbi WA, Chambers JB, Dumesnil JG, Foster E, Gottdiener JS, Grayburn PA, et al. Recommendations for evaluation of prosthetic valves with echocardiography and Doppler ultrasound: a report From the American Society of Echocardiography's Guidelines and Standards Committee and the Task Force on Prosthetic Valves, developed in conjunction with the American College of Cardiology Cardiovascular Imaging Committee, Cardiac Imaging Committee of the American Heart Association, the European Association of Echocardiography, a registered branch of the European Society of Cardiology, the Japanese Society of Echocardiography and the Canadian Society of Echocardiography, endorsed by the American College of Cardiology Foundation, American Heart Association, European Association of Echocardiography, a registered branch of the European Society of Cardiology, the Japanese Society of Echocardiography, and Canadian Society of Echocardiography. J Am Soc Echocardiogr. 2009;22:975-1014.

7. Pislaru SV, Hussain I, Pellikka PA, Maleszewski JJ, Hanna RD, Schaff HV, et al. Misconceptions, diagnostic challenges and treatment opportunities in bioprosthetic valve thrombosis: lessons from a case series. Eur J Cardiothoracic Surg. 2015;47:725-32.

8. Oliver JM, Gallego P, Gonzalez A, Dominguez FJ, Gamallo C, Mesa JM. Bioprosthetic mitral valve thrombosis: clinical profile, transesophageal echocardiographic features, and follow-up after anticoagulant therapy. J Am Soc Echocardiogr. 1996;9:691-9.

9. Butnaru A, Shaheen J, Tzivoni D, Tauber R, Bitran D, Silberman S. Diagnosis and treatment of early bioprosthetic malfunction in the mitral valve position due to thrombus formation. Am J Cardiol. 2013;112:1439-44.

10. De Marchena E, Mesa J, Pomenti S, Marin Y, Kall C, Marincic X, et al. Thrombus formation following transcatheter aortic valve replacement. JACC Cardiovasc Interv. 2015;8:728-39.

11. Latib A, Naganuma T, Abdel-Wahab M, Danenberg H, Cota L, Barbanti M, et al Treatment and clinical outcomes of transcatheter heart valve thrombosis. Circ Cardiovasc Interv. 2015;8:e01779.

12. Nishimura RA, Otto CM, Bonow RO, Carabello BA, Erwin JP III, Guyton RA, et al. 2014 AHA/ACC guideline for the management of patients with valvular 
heart disease: executive summary. A report of the American College of Cardiology/American Heart Association Task Force on Practice Guidelines. Circulation. 2014;129:2440-92.

13. Vahanian A, Alfieri O, Andreotti F, Antunes MJ, Barón-Esquivias G, Baumgartner $\mathrm{H}$, et al. Guidelines on the management of valvular heart disease (version 2012): the Joint Task Force on the Management of Valvular Heart Disease of the European Society of Cardiology (ESC) and the European Association for Cardio-Thoracic Surgery (EACTS). Eur J Cardiothoracic Surg. 2012;42:S1-44.

14. Mérie C, Kober L, Skov Olsen P, Andersson C, Gislason G, Skov Jensen J, et al. Association of warfarin therapy duration after bioprosthetic aortic valve replace- ment with risk of mortality, thromboembolic complications, and bleeding. JAMA 2012;308:2118-25.

15. Brown ML, Park SJ, Sundt TM, Schaff HV. Early thrombosis risk in patients with biologic valves in the aortic position. J Thorac Cardiovasc Surg. 2012;144: $108-11$.

Key Words: bioprosthetic valve, thrombosis, echocardiography

\title{
EDITORIAL COMMENTARY
}

\section{Bioprosthetic valve thrombosis: Are we not seeing the wood for the trees?}

\author{
Clifford W. Barlow, DPhil, FRCS(CTh) \\ From the Department of Cardiothoracic Surgery, University Hospital Southampton, Southampton, United \\ Kingdom. \\ Disclosures: Author has nothing to disclose with regard to commercial support. \\ Received for publication July 26, 2016; accepted for publication July 26, 2016. \\ Address for reprints: Clifford W. Barlow, DPhil, FRCS(CTh), Department of Cardiothoracic Surgery, University \\ Hospital Southampton, Tremona Rd, Southampton SO16 6YD, United Kingdom (E-mail: cliffbarlow@ \\ hotmail.com or clifford.barlow@uhs.nhs.uk). \\ J Thorac Cardiovasc Surg 2016;152:978-80 \\ $0022-5223 / \$ 36.00$ \\ Copyright $(\underset{2016}{ } 20 \mathrm{by}$ The American Association for Thoracic Surgery \\ http://dx.doi.org/10.1016/j.jtcvs.2016.07.059
}

A major decision facing many patients undergoing valve replacement is selection of valve prosthesis, traditionally assessing the risks of lifelong anticoagulation after mechanical valve replacement versus the risks of bioprosthetic valve degeneration and possible reoperation. Despite promising improvements in the longevity of newer-generation bioprosthetic valves, reduced risks associated with redo surgery, and now the possibility of redo "valve-in-valve" transcatheter aortic valve replacement (TAVR), improving outcomes after bioprosthetic valve replacement remains important.

In this edition of the Journal, there is a provocative Expert Opinion by Egbe and the group ${ }^{1}$ from the Mayo Clinic, on an underrecognized problem: bioprosthetic valve thrombosis (BPVT). On the basis of their own study, ${ }^{2}$ the authors clearly explain how it may be the presence of thrombus on bioprosthetic valves, and not valve degeneration, that causes reduced leaflet motion, a resultant increase in transvalvar gradients, and possible hemodynamic compromise.

Egbe and colleagues' study ${ }^{2}$ of 397 valve explants found pathologic evidence of BPVT in 46 cases $(11 \%)$, with $65 \%$ of these occurring more than 1 year after valve insertion and $15 \%$ after 5 years, suggesting this is not a

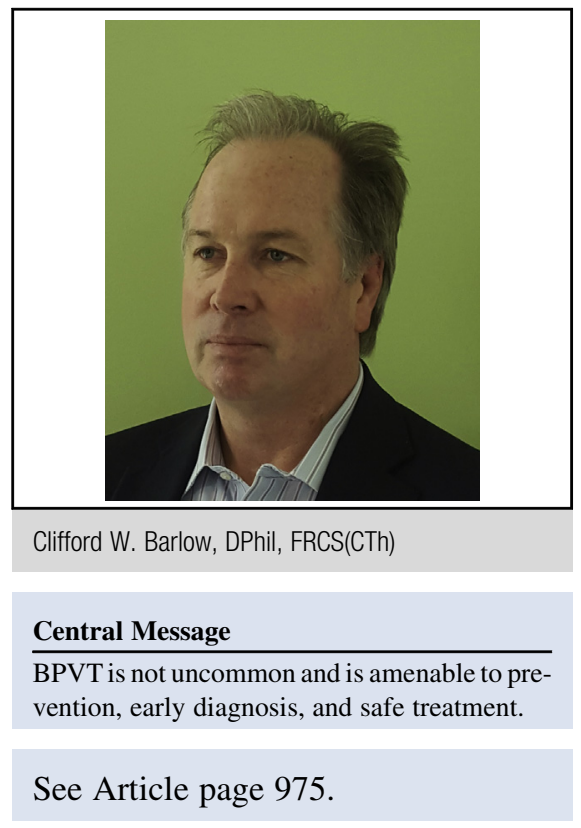

perioperative phenomenon. By using echocardiographic criteria, particularly increased cusp thickness, decreased cusp mobility, and increasing transvalvar gradients, they estimated the incidence of BPVT to be at least $1 \%$, based on 3161 patients who underwent follow-up echocardiograms at their institution. They demonstrate the efficacy of warfarin therapy in treating BPVT in 14 of 15 cases of established BPVT (93\%), thereby avoiding reoperation. ${ }^{3}$ They also consider that antiplatelet therapy, or guideline-directed ${ }^{4,5}$ (3 months only) anticoagulation, is ineffective in preventing BPVT. Finally, they reference the recent New England Journal of Medicine 